\title{
Therapeutic Effects of Chinese Herbal Formula (PTQX) on NC/Nga Mice with Atopic Dermatitis-Like Skin Lesions
}

\author{
Fenggen Yan, ${ }^{1}$ Jing Zhang, ${ }^{1}$ Xiong Li, ${ }^{1}$ Xiumei Mo, ${ }^{1}$ Junfeng Liu, ${ }^{1}$ Siqi Ye, ${ }^{1}$ Ying Lin, ${ }^{1}$ \\ Xiaohui Qiu, ${ }^{1}$ Simon Mingyuen Lee $\mathbb{D}^{2}{ }^{2}$ and Dacan Chen $\mathbb{D i D}^{1}$ \\ ${ }^{1}$ Department of Dermatology, The Second Affiliated Hospital of Guangzhou University of Chinese Medicine, \\ Guangdong Provincial Hospital of Chinese Medicine, Guangdong Provincial Key Laboratory of Chinese Medicine for \\ Prevention and Treatment of Refractory Chronic Diseases, Guangzhou, Guangdong, China \\ ${ }^{2}$ State Key Laboratory of Quality Research in Chinese Medicine and Institute of Chinese Medical Sciences, University of Macau, \\ Macau, China \\ Correspondence should be addressed to Dacan Chen; cdc@gzucm.edu.cn
}

Received 27 June 2019; Accepted 4 August 2019; Published 7 December 2019

Academic Editor: Simona Martinotti

Copyright (C) 2019 Fenggen Yan et al. This is an open access article distributed under the Creative Commons Attribution License, which permits unrestricted use, distribution, and reproduction in any medium, provided the original work is properly cited.

\begin{abstract}
Atopic dermatitis (AD), also known as atopic eczema, is a chronic pruritic inflammatory skin disease. The available systemic therapies for atopic dermatitis are inadequate. Objective. This study aimed to evaluate the effects of the Chinese herbal formula Pei Tu Qing Xin (PTQX) on dermatitis severity and ear swelling, immunomodulation, and the infiltration of mast cells in a mouse model of 1-chloro-2,4-dinitrobenzene- (DNCB-) induced AD. Methods. AD-like symptoms were induced by DNCB in NC/Nga mice. Skin lesions, dermatitis, ear swelling, and scratching behaviour were evaluated. Changes in the T-helper type 1 (Th1), Th2, Th17, and regulatory $\mathrm{T}$ (Treg) subtypes and immunoregulation in the spleen and lymph nodes were detected by flow cytometry. Results. Histopathological and immunohistochemical analyses demonstrated that PTQX decreased the DNCB-mediated induction of mast cells and infiltration of inflammatory cells in the ear and dorsal skin. PTQX also reduced the DNCB-induced increase in the serum immunoglobulin E level, pruritus, and dermatitis (red, flaky areas) on the dorsal skin. Furthermore, PTQX regulated the balance between the populations of Th1, Th2, Th17, and Treg cells (particularly the latter two) in the lymph nodes. Conclusions. Our results suggest that the Chinese herbal formula PTQX can alleviate symptoms of AD, such as epithelial damage, redness, swelling, and pruritus, and potentially be used to treat this condition.
\end{abstract}

\section{Introduction}

Atopic dermatitis (AD) is a chronic, highly treatment-refractory form of itchy inflammatory skin dermatosis that affects both children and adults. It is among the most common chronic skin diseases, affecting up to $25 \%$ of children and $2-3 \%$ of adults in most countries worldwide [1]. Clinically, $\mathrm{AD}$ is a chronic form of pruritic inflammatory skin disease characterised by red, burning, dry, swollen, and cracked skin. The pathogenesis of AD is complex and involves multiple contributors such as skin barrier defects, infections, immunological factors (T-helper (Th) cells, inflammatory cells, immunoglobulin E, cytokines, and chemokines), susceptible genes (filaggrin genes), and environmental interactions (food and aeroallergens and seasonal and climatic changes) [2-4]. Currently, no treatment can effectively cure or control the recurrence of AD. No standardised international guidelines have been set for $\mathrm{AD}$, and the internally and topically administered hormone or immune inhibitors and antihistamines used in traditional Western medicine elicit side effects and are not ideal. Therefore, alternative medicinal treatments for $\mathrm{AD}$, including traditional Chinese medicine (TCM), have elicited broad and increasing interest in recent years $[5,6]$.

TCM has been used clinically for thousands of years, including the routine management and treatment of $\mathrm{AD}$, 
and its efficacy has been proven through scientific investigation [7]. A recent expert consensus recommended the TCM formula Pei Tu Qing Xin (PTQX) as a prescribed treatment for $\mathrm{AD}$ [8]. Previously, our team reported an outcome assessor-blinded, placebo-controlled, 3-arm randomised clinical trial (RCT) conducted to evaluate the efficacy and safety of PTQX for the treatment of AD. In our study, PTQX effectively relieved the symptoms of $\mathrm{AD}$ and improved the self-assessed quality of life (QOL) in patients with moderate to severe $\mathrm{AD}$, with no reported severe adverse events [9]. Furthermore, a study protocol for a RCT intended to test the efficacy and safety of oral PTQX in children aged 6-16 years with moderate-to-severe atopic eczema has been published in trials [10]. However, the molecular mechanisms underlying the effects of PTQX on $\mathrm{AD}$ remain unclear.

Our previous study indicated that PTQX considerably relieved the symptom of pruritus in a guinea pig model by reducing the release of endogenous and exogenous histamine [11]. The anti-inflammatory effects of PTQX were assessed in 3 different animal models: xylene-induced oedema in the mouse ear, cotton pellet granuloma formation, and an acetic acid-induced increase in abdominal cavity capillary permeability [12]. A further investigation focused on the effects of PTQX on Th1 and Th2 cell differentiation in the spleens of C57BL/6 mice with induced AD. Compared with the model group, mice in the PTQX group exhibited reduced inflammation and regulation of the Th1/Th2 cell balance [13].

This study aimed to evaluate the therapeutic effects of PTQX on 1-chloro-2,4-dinitrobenzene- (DNCB-) induced AD-like symptoms in NC/Nga mice. We demonstrate here that PTQX can improve the symptoms of AD and reduce the inflammatory infiltration of CD4+ and CD8+ T cells into DNCB-induced skin lesions in these mice. We further evaluated the anti-inflammatory and antiallergic properties of PTQX in vivo and found that this treatment inhibited the total production of immunoglobulin $\mathrm{E}$ ( $\mathrm{IgE}$ ) as measured in serum, suppressed ear swelling, and significantly alleviated scratching behaviour. Furthermore, our experimental results demonstrate that PTQX inhibited the infiltration of mast and other inflammatory cells in skin lesions and regulated the Th/regulatory $\mathrm{T}$ (Treg) cell balance in lymphoid organs. Our findings suggest the potential therapeutic effects of the Chinese herbal formula PTQX against DNCB-induced ADlike skin lesions in NC/Nga mice.

\section{Materials and Methods}

2.1. Chemicals, Reagents, and Materials. High-performance liquid chromatography (HPLC) grade acetonitrile, methanol, and formic acid were obtained from Sigma-Aldrich (St. Louis, MO, USA). Ultrapure water was prepared using a Milli-Q water system (Millipore, Billerica, MA, USA). Chemical references, including liquiritin, liquirtigenin, isoliquiritin, isoliquiritigenin, and glycyrrhizin, were purchased from Chengdu Must Bio-Technology Co., Ltd. (Chengdu, China). Isoforsythiaside A, forsythoside A, and forsythin were obtained from Shanghai YuanYe Biotechnology Co., Ltd. (Shanghai, China). Chlorogenic acid, forsythoside $\mathrm{B}$, and obacunone were obtained from the
National Institutes for Food and Drug Control (Beijing, China). All standards were determined to have a purity of $\geq 95 \%$ by HPLC. All references were deliquated with methanol to a concentration of $50.0 \mu \mathrm{g} / \mathrm{mL}$. PTQX granules were produced on a pilot-scale by Jiangyin Tian Jiang Pharmaceutical Co., Ltd. (Jiangsu, China).

DNCB (1-chloro-2,4-dinitrobenzene) and enzymelinked immunosorbent assay (ELISA) kits for IgE were obtained from Sigma-Aldrich. Mouse Th1/Th2/Th17 and Th17/Treg phenotyping kits were obtained from BD Biosciences (San Jose, CA, USA). All other chemicals and solvents were of the highest commercially available grade.

2.2. Chemical Constitution Analysis of PTQX Using UltraHPLC- (UHPLC-) LTQ-Orbitrap-Mass Spectrometry (MS). The Chinese herbal formula PTQX was prepared in TCM granule form using extracts from 9 Chinese medicinal substances, including Rhizoma Atractylodis Macrocephalae (Bai Zhu) 10 g, Radix Pseudostellariae (Tai Zi Shen) 10 g, Rhizoma Dioscoreae (Shan Yao) $15 \mathrm{~g}$, Semen Coicis (Yi Yi Ren) 20 g, Rhizoma Imperatae (Bai Mao Gen) 15 g, Fructus Forsythiae (Lian Qiao) $10 \mathrm{~g}$, Cortex Dictamni (Bai Xian Pi) $10 \mathrm{~g}$, Margarita (Zhen Zhu Feng) $0.3 \mathrm{~g}$, and Radix Glycyrrhizae (Gan Cao) $5 \mathrm{~g}$ [10]. This formula has been patented for the treatment of $\mathrm{AD}$ (patent no.: ZL2013 1 0328668.4, China).

UHPLC-LTQ-Orbitrap-MS was used to specify the chemical compounds present in PTQX granules. Chromatographic separation was conducted on an Accela UHPLC system (Thermo Fisher Scientific, San Jose, CA, USA). The sample solution was filtered and analysed on a Phenomenex Kinetex $\mathrm{C}_{18}$ column $(2.1 \mathrm{~mm} \times 100 \mathrm{~mm}$ i.d., $1.7 \mu \mathrm{m})$. The mobile phase comprised a mixture of $0.1 \%$ formic acid (A) and acetonitrile (B). The elution gradient was as follows: $0-20 \mathrm{~min}, 8-18 \% \mathrm{~B} ; 20-25 \mathrm{~min}, 18-40 \% \mathrm{~B}$; and $25-32 \mathrm{~min}, 40-85 \% \mathrm{~B}$. The injection volume was $2 \mu \mathrm{L}$, and the flow rate was $300 \mu \mathrm{L} / \mathrm{min}$. Since flavonoids, phenethyl alcohol glycosides, lignans, saponins, and limonoids were the major structural types mainly detected and identified in the PTQX formula, they might be the main contributions for the AD-related activities. For instance, it has been reported that rutin (compound 16) could be a potential therapeutic agent for the treatment of $\mathrm{AD}$ and allergic contact dermatitis [14]. In addition, a previous study showed that isoliquiritigenin (compound 28) has therapeutic effects in the treatment of atopic dermatitis-like skin lesions in mice by inhibiting p38 and ERK activation [15]. Furthermore, glycyrrhizin (compound 29) can also alleviate atopic dermatitis-like symptoms [16].

For the qualitative analysis, an LTQ-Orbitrap XL mass spectrometer was coupled with the LC device via an electrospray ionisation (ESI) interface (Thermo Fisher Scientific, Bremen, Germany). The samples were determined in the negative mode using the following ESI parameters: spray voltage, $-3.3 \mathrm{kV}$; capillary temperature, $325^{\circ} \mathrm{C}$; tube lens voltage, $-72 \mathrm{~V}$; sheath gas, 45 units; and auxiliary gases, 6 units. The Orbitrap mass analyser was set to the full scan 
mass range with an $\mathrm{m} / z$ ratio of $110-1100$ and resolution of 30,000. The following limits for the potentially expected atoms in the components were set: carbon $\leq 50$, hydrogen $\leq 80$, oxygen $\leq 30$, and nitrogen $\leq 9$. The accuracy error threshold was set at $5 \mathrm{ppm}$. Xcalibur 2.1 software (Thermo Fisher Scientific) was used to analyse the data. The chemical compounds in PTQX granules were distinguished through comparisons with mass data from the reference standards or fragment information in the literature.

2.3. Mouse Model of DNCB-Induced AD. Specific pathogenfree male NC/Nga mice (age: 6-8 weeks) were obtained from the Riken BioResource Centre in Japan. The animals were kept in a room with a controlled temperature of $22 \pm 3^{\circ} \mathrm{C}$, relative humidity of $55 \pm 5 \%$, and illumination with a $12-\mathrm{h}$ light/dark cycle for at least 1 week prior to the experiments. All animals were fed with standard rodent chow daily and fresh tap water ad libitum. All animal experiments were performed under anaesthesia. Ten grams of 2,2,2-tribromoethanol (Avertin, Sigma-Aldrich, USA) was dissolved in $10 \mathrm{ml}$ of tertamyl alcohol to yield a stock solution. A final working solution was prepared by diluting $1 \mathrm{ml}$ of stock solution with $39 \mathrm{ml}$ of isotonic saline. For anaesthesia, the stock solution was administered intraperitoneally (i.p.; $12-15 \mu \mathrm{l} / \mathrm{g}$ of animal body weight). All animal care, use, and experimental procedures were conducted in accordance with the Laboratory Animal Research Committee Guidelines of Guangdong Provincial Hospital of Chinese Medicine (approval number 2016015).

The mice were randomly divided into four experimental groups ( $n=7-8$ per group). AD-like immunological and skin lesions were induced in NC/Nga mice by treatment with DNCB as described previously, with minor modifications [17]. Briefly, DNCB was applied to a patch of dorsal skin, the face, and the back of both ears of each mouse. After the complete removal of dorsal hair from an approximately $8 \mathrm{~cm}^{2}$ area, AD-like skin lesions were topically induced by sensitisation with $200 \mu \mathrm{l}$ of $1 \%$ DNCB dissolved in acetoneolive oil (AO, $3: 1$ ). Additionally, $20-\mu$ l aliquots of this $1 \%$ DNCB working solution were applied repeatedly to the face and the backs of both ears on days -4 and 0 . All mice except those in the control group were challenged with $0.2 \%$ DNCB applied thrice weekly for 3 weeks (days 1-20) on the same areas of the skin to induce $\mathrm{AD}$-like lesions. On day 21, the mice were sacrificed under anaesthesia as described above and samples were collected for further analyses. Mice in the control group were treated with vehicle.

For treatment, PTQX was suspended in pure water and administered orally at a volume of $20 \mathrm{ml} / \mathrm{kg}$ to the PTQXtreated group $(8.0 \mathrm{~g} / \mathrm{kg})$ every day since the first sensitisation. A dexamethasone-treated group $(10 \mathrm{ml} / \mathrm{kg}$, daily oral $3.0 \mathrm{mg} / \mathrm{kg}$ ) was maintained as the positive control. The model group received the same volume of pure water. The experimental design is summarised in Figure 1.

2.4. Evaluation of Dermatitis Severity and Ear Thickness. After anesthetising mice with 2,2,2-tribromoethanol, the skin lesions were imaged using a digital camera (Canon,
Tokyo, Japan) on day 7 of the experiment. We evaluated the dorsal dermatitis scores once weekly according to the previously published standards $[17,18]$. The severity of AD was graded on the following scale: $0=$ none, $1=$ mild, $2=$ moderate, and $3=$ severe. This scale was applied to each of the four symptoms: (i) erythema/haemorrhage, (ii) oedema, (iii) excoriation/erosion, and (iv) scaling/dryness. The total dermatitis scores can range from 0 to 12 points. We further measured the thicknesses of the right and left auricles weekly during the study period using an electronic digital calliper (Guangxi, China). To minimise technical variation, a single investigator performed all measurements during each experiment.

2.5. Evaluation of Scratching Behaviour. NC/Nga mice with AD-like symptoms exhibit pathological and behavioural characteristics very similar to those observed in patients with AD. For elucidation, the duration of scratching behaviour was defined as the time spent rubbing the head and scratching the dorsal skin, nose, and face with the hind limbs. A scratching behaviour event was defined as a single 20-minute incidence of scratching and was measured using a digital camera facing the test box on the penultimate day of the experiment (day 20).

2.6. Histology and Immunohistochemistry (IHC). Histological and immunohistochemical changes can reflect pathological changes in the tissue sections. For comparison, samples of the dorsal skin and one ear were collected from each mouse on the last day of the experiment. The tissues were stored in cold 4\% paraformaldehyde overnight and embedded in paraffin wax according to the standard procedures. Subsequently, the tissues were sectioned at a thickness of $4 \mu \mathrm{m}$ and mounted on silane-coated glass slides. Deparaffinised and rehydrated tissue sections were stained with haematoxylin and eosin ( $\mathrm{HE}$ ) or toluidine blue (TB) to detect infiltrating inflammatory cells or mast cells, respectively. To characterise the infiltrating $\mathrm{CD} 4+$ and CD8+ lymphocytes in ear tissues, primary monoclonal rat antibodies against mouse CD8a (clone 53-6.7) and CD4 (GK1.5) were used to indirectly stain the samples. Subsequently, a horseradish peroxidase-(HRP-)conjugated goat anti-mouse IgG-HRP secondary antibody was used to label the bound primary antibodies. Tissue sections were examined using an Olympus BX53 light microscope (Tokyo, Japan).

2.7. Flow Cytometry Analysis. Cell suspensions were isolated from the draining lymph nodes of the mice in each group. Suspensions of the mouse spleen and lymph node cells (10 million/ml in media) were activated for 5 hours using phorbol-12-myristate-13-acetate (PMA) and ionomycin (50 $\mathrm{ng} / \mathrm{ml}$ and $1 \mu \mathrm{g} / \mathrm{ml}$, respectively) in the presence of $\mathrm{BD}$ GolgiStop $^{\mathrm{TM}}$ Protein Transport Inhibitor according to the manufacturer's instructions. Flow cytometry was performed on a BD FACSAria ${ }^{\mathrm{TM}}$ III device. 


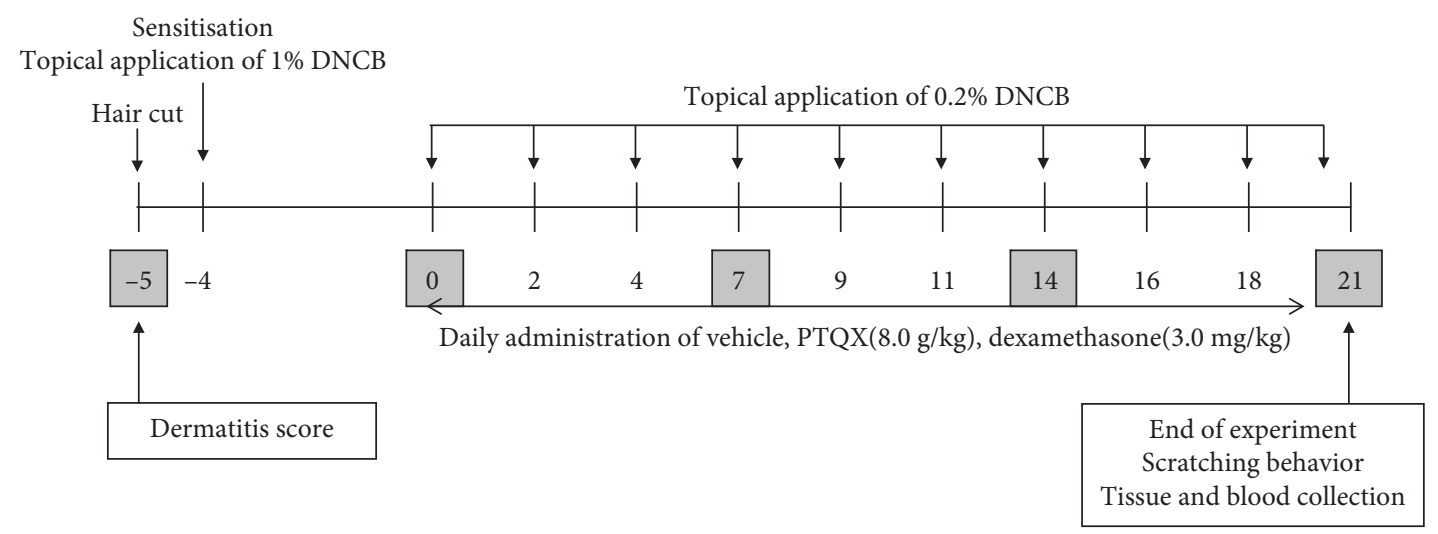

FIGURE 1: Experimental scheme for the 1-chloro-2,4-dinitrobenzene- (DNCB-) induced model of atopic dermatitis (AD).

2.8. Total Serum IgE Levels. At the end of the experiment, blood samples were collected from the mice and serum samples were obtained by centrifugation $(3500 \mathrm{rpm}$ for $15 \mathrm{~min}$ ) and stored at $-80^{\circ} \mathrm{C}$ until use. The total serum $\operatorname{IgE}$ levels were measured using a mouse IgE ELISA kit (SigmaAldrich) according to the manufacturer's instructions.

2.9. Statistical Analyses. Data were expressed as mean$s \pm$ standard errors of the means (SEM). A one-way analysis of variance (ANOVA) or Student's $t$-test was used to determine the significance of differences. $P$ values of $<0.05$ and $<0.01$ were considered significant.

\section{Results}

3.1. Quality Control Analysis of PTQX. The phytochemical constituents in an extract of PTQX granules were identified by UHPLC-LTQ-Orbitrap-MS in the ESI negative-ion mode. A base peak chromatogram of the PTQX granule extract was acquired for structural confirmation (Figure 2). The authentic compounds and their MS/MS fragmentation behaviours were conducted to elucidate the chemical components. Thirty-two components were identified in the PTQX granules through comparisons with the retention and mass behaviours of standards or data in the literature [19-21]. Specifically, the major constituents of the PTQX extract were attributed to Forsythiae Fructus (18 compounds), Glycyrrhizae Radix et Rhizoma (9 compounds), and Dictamni Cortex (4 compounds). The quasimolecular ions of corresponding compounds and their fragment ions in the MS/MS spectra are listed in Table 1.

\subsection{PTQX Attenuated DNCB-Induced AD-Like Symptoms.} We examined whether PTQX could inhibit DNCB-induced AD-like skin inflammation in mice. Representative dorsal skin photographs, dermatitis scores, and ear thicknesses were obtained from mice treated or not with PTQX to compare $\mathrm{AD}$-like skin lesions. In $\mathrm{AD}$ mice, the dorsal skin exhibited severe erythema, erosion, and dryness which was ameliorated by treatment with PTQX (Figure 3(a)). In addition, the dermatitis score was significantly higher in the vehicle-treated group compared with the control group. We further observed that treatment with PTQX or DEX significantly ameliorated the severity of DNCB-induced increases in the dermatitis score (Figure $3(\mathrm{~b})$ ). At the end of the experiment (day 21), a measurement of the treated mouse ears revealed that PTQX significantly inhibited the DNCB-induced increase in ear thickness (Figure 3(c)). In other words, the gradual ear oedema induced in $\mathrm{NC} / \mathrm{Nga}$ mice by DNCB was clearly and similarly inhibited by both DEX and PTQX (Figure 3(d)).

3.3. PTQX Decreased Serum IgE Levels. A close correlation has been observed between serum IgE levels and AD. The high IgE levels characteristic of $\mathrm{AD}$ place the patient at risk of sensitisation to food allergens and aeroallergens. Therefore, the total serum IgE level is an important indicator in AD. We used an ELISA to detect the total serum IgE levels in the mice from each group. We observed a marked increase in this indicator in the vehicle group, compared with the normal control group $(37760 \pm 6971$ vs. $318.9 \pm 59.3 \mathrm{ng} / \mathrm{mL})$. Compared to the vehicle, PTQX significantly inhibited this increase in the total serum IgE level $(14145 \pm 3457 \mathrm{ng} / \mathrm{mL})$, suggesting that this TCM formula could suppress the IgE synthesis associated with AD. Treatment with DEX also clearly and significantly depressed the increase in total serum IgE levels (Figure 3(e)).

\subsection{PTQX Inhibits Mast Cell Infiltration and Scratching} Frequency in NC/Nga Mice. Mast cells have long been recognised as the major effector cells in the pathogenesis of $\mathrm{AD}[22,23]$. To determine whether PTQX reduces the infiltration of mast cells into the skin, we stained skin samples with toluidine blue. Larger numbers of mast cells were detected in the dermis of the AD model mice, compared to the normal mice (Figure 4(a)). Sensitisation with DNCB significantly increased the infiltration of mast cells into the dorsal skin lesions $(34.5 \pm 1.96)$. Treatment with DEX significantly inhibited this infiltration $(24.67 \pm 2.843)$. Similarly, treatment with PTQX also reduced the number of infiltrating mast cells $(18.44 \pm 3.469)$ (Figure 4(b)).

The severe itching and scratching associated with $\mathrm{AD}$ not only affect a patient's quality of life, but also exacerbate disease progression. Therefore, an analysis of spontaneous 


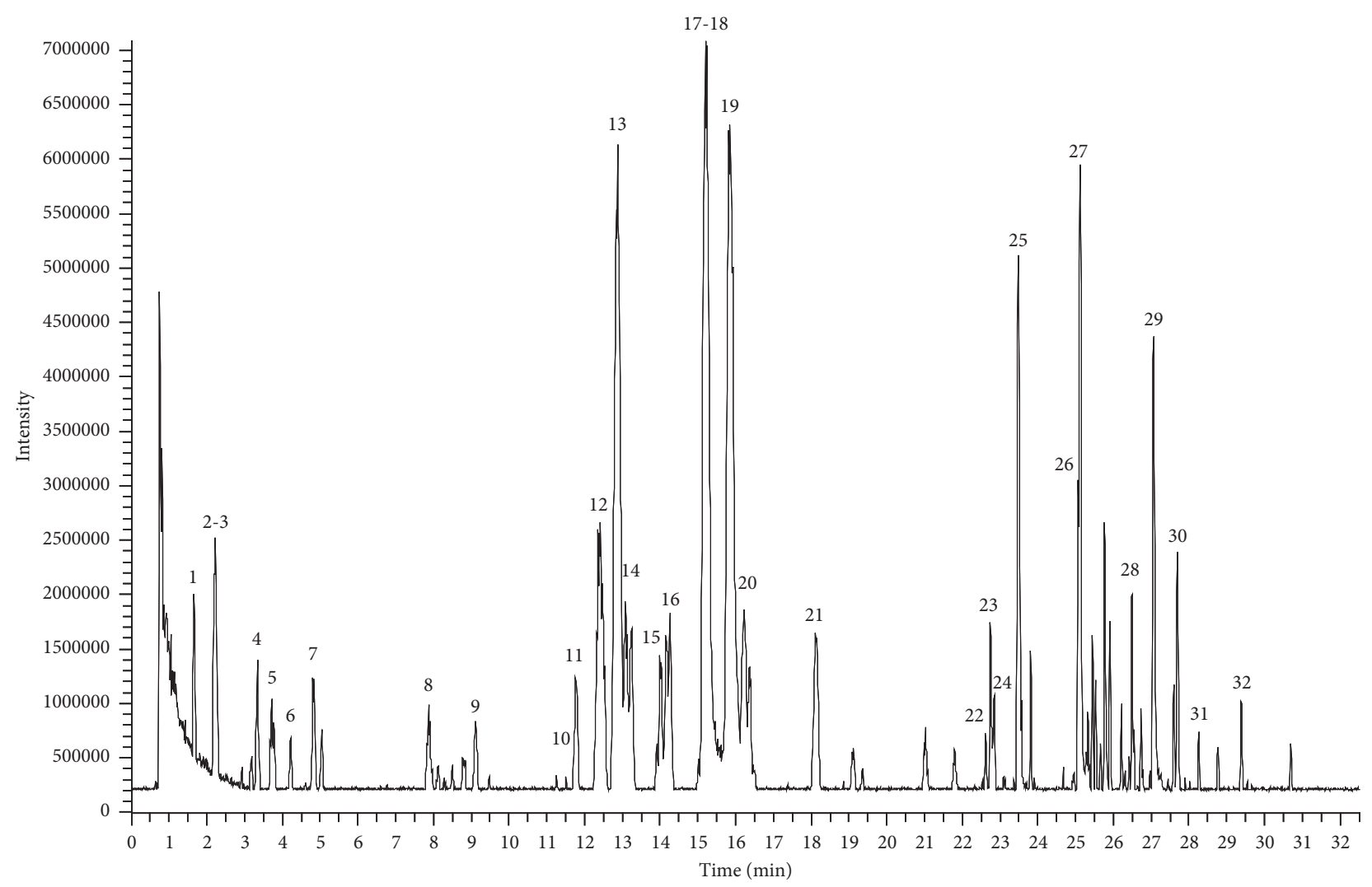

FIGURE 2: Liquid chromatography- (LC-) mass spectrometry (MS) base peak chromatogram of an extract of PTQX.

TABLE 1: Compounds detected and identified in PTQX formula.

\begin{tabular}{|c|c|c|c|c|c|}
\hline No. & $t_{\mathrm{R}}(\min )$ & {$[\mathrm{M}-\mathrm{H}]^{-}$or $\left[\mathrm{M}+\mathrm{COO}^{-}\right]^{-}(\mathrm{ppm})$} & Identification & Formula & Resource \\
\hline 1. & 1.66 & $375.12930(0.73)$ & Adoxosidic acid & $\mathrm{C}_{16} \mathrm{H}_{24} \mathrm{O}_{10}$ & $\mathrm{Ff}$ \\
\hline 2. & 2.16 & $353.08701(0.30)$ & Neochlorogenic acid & $\mathrm{C}_{16} \mathrm{H}_{18} \mathrm{O}_{9}$ & Ir, Am, Ff \\
\hline 3. & 2.23 & $461.16556(0.21)$ & Forsythoside E & $\mathrm{C}_{20} \mathrm{H}_{30} \mathrm{O}_{12}$ & $\mathrm{Ff}$ \\
\hline 4. & 3.34 & $209.04535(0.35)$ & Unknown & $\mathrm{C}_{10} \mathrm{H}_{10} \mathrm{O}_{5}$ & Grr \\
\hline 5. & 3.71 & $353.08701(0.39)$ & Chlorogenic acid ${ }^{\mathrm{b}}$ & $\mathrm{C}_{16} \mathrm{H}_{18} \mathrm{O}_{9}$ & $\mathrm{Ir}, \mathrm{Am}, \mathrm{Ff}$ \\
\hline 6. & 4.22 & $353.08682(0.11)$ & Cryptochlorogenic acid & $\mathrm{C}_{16} \mathrm{H}_{18} \mathrm{O}_{9}$ & Ir, Am, Ff \\
\hline 7. & 4.80 & $179.03476(-0.46)$ & Caffeic acid & $\mathrm{C}_{9} \mathrm{H}_{8} \mathrm{O}_{4}$ & $\mathrm{Ff}, \mathrm{Ir}$ \\
\hline 8. & 7.85 & $229.10760(0.55)$ & Unknown & $\mathrm{C}_{11} \mathrm{H}_{18} \mathrm{O}_{5}$ & Dc \\
\hline 9. & 9.11 & $495.14914(-0.56)$ & Forsythenside $\mathrm{A}^{\mathrm{b}}$ & $\mathrm{C}_{22} \mathrm{H}_{26} \mathrm{O}_{10}$ & $\mathrm{Ff}$ \\
\hline 10. & 11.25 & $581.18518^{\mathrm{a}}(-1.30)$ & Forsythiaside B & $\mathrm{C}_{26} \mathrm{H}_{32} \mathrm{O}_{12}$ & $\mathrm{Ff}$ \\
\hline 11. & 11.76 & $609.18066(-0.74)$ & Calceolarioside $\mathrm{C} /$ lianqiaoxinoside $\mathrm{C}$ & $\mathrm{C}_{28} \mathrm{H}_{34} \mathrm{O}_{15}$ & $\mathrm{Ff}$ \\
\hline 12. & 12.42 & $417.11813(0.12)$ & Liquiritin $^{\mathrm{b}}$ & $\mathrm{C}_{21} \mathrm{H}_{22} \mathrm{O}_{9}$ & Grr \\
\hline 13. & 12.86 & $623.19684(-0.21)$ & Isoforsythiaside $\mathrm{A}^{\mathrm{b}}$ & $\mathrm{C}_{29} \mathrm{H}_{36} \mathrm{O}_{15}$ & $\mathrm{Ff}$ \\
\hline 14. & 13.08 & $549.16028(0.01)$ & Licuroside & $\mathrm{C}_{26} \mathrm{H}_{30} \mathrm{O}_{13}$ & Grr \\
\hline 15. & 14.01 & $609.18097(-0.98)$ & Calceolarioside $\mathrm{C} /$ lianqiaoxinoside $\mathrm{C}$ & $\mathrm{C}_{28} \mathrm{H}_{34} \mathrm{O}_{15}$ & $\mathrm{Ff}$ \\
\hline 16. & 14.17 & $609.14447(-1.09)$ & Rutin & $\mathrm{C}_{27} \mathrm{H}_{30} \mathrm{O}_{16}$ & $\mathrm{Ff}$ \\
\hline 17. & 15.01 & $527.21161^{\mathrm{a}}(-0.69)$ & Forsythenside G & $\mathrm{C}_{24} \mathrm{H}_{34} \mathrm{O}_{10}$ & $\mathrm{Ff}$ \\
\hline 18. & 15.21 & $623.19702(-0.03)$ & Forsythoside $\mathrm{A}^{\mathrm{b}}$ & $\mathrm{C}_{29} \mathrm{H}_{36} \mathrm{O}_{15}$ & $\mathrm{Ff}$ \\
\hline 19. & 15.70 & $755.23737(-1.94)$ & Forsythoside $\mathrm{B}^{\mathrm{b}}$ & $\mathrm{C}_{34} \mathrm{H}_{44} \mathrm{O}_{19}$ & Ff \\
\hline 20. & 16.19 & $519.18616(-0.48)$ & Epipinoresinol-4-O- $\beta$-D-glucopyranoside & $\mathrm{C}_{36} \mathrm{H}_{32} \mathrm{O}_{11}$ & Ff \\
\hline 21. & 18.11 & $565.19086(-1.26)$ & Pinoresinol-4-O- $\beta$-D-glucopyranoside & $\mathrm{C}_{36} \mathrm{H}_{32} \mathrm{O}_{11}$ & $\mathrm{Ff}$ \\
\hline 12. & 21.79 & $475.12305^{\mathrm{a}}(0.5)$ & Ononin & $\mathrm{C}_{22} \mathrm{H}_{22} \mathrm{O}_{9}$ & Grr \\
\hline 23. & 22.83 & $417.11844(0.43)$ & Isoliquiritin $^{\mathrm{b}}$ & $\mathrm{C}_{21} \mathrm{H}_{22} \mathrm{O}_{9}$ & Grr \\
\hline 24. & 23.01 & $255.06586(0.68)$ & Liquirtigenin $^{\mathrm{b}}$ & $\mathrm{C}_{15} \mathrm{H}_{12} \mathrm{O}_{4}$ & Grr \\
\hline 25. & 23.46 & $579.20715^{\mathrm{a}}(-0.07)$ & Forsythin ${ }^{b}$ & $\mathrm{C}_{27} \mathrm{H}_{34} \mathrm{O}_{11}$ & $\mathrm{Ff}$ \\
\hline 26. & 25.05 & $1011.42535(-0.73)$ & Unknown & $\mathrm{C}_{70} \mathrm{H}_{60} \mathrm{O}_{7}$ & Grr \\
\hline 27. & 25.10 & $849.37482(-0.80)$ & Unknown & $\mathrm{C}_{39} \mathrm{H}_{62} \mathrm{O}_{20}$ & Grr \\
\hline
\end{tabular}


TABle 1: Continued.

\begin{tabular}{|c|c|c|c|c|c|}
\hline No. & $t_{\mathrm{R}}(\min )$ & {$[\mathrm{M}-\mathrm{H}]^{-}$or $\left[\mathrm{M}+\mathrm{COO}^{-}\right]^{-}(\mathrm{ppm})$} & Identification & Formula & Resource \\
\hline 28. & 26.73 & $255.06592(0.74)$ & Isoliquiritigenin $^{\mathrm{b}}$ & $\mathrm{C}_{15} \mathrm{H}_{12} \mathrm{O}_{4}$ & Grr \\
\hline 29. & 27.06 & $821.39496(-0.45)$ & Glycyrrhizin ${ }^{\mathrm{b}}$ & $\mathrm{C}_{42} \mathrm{H}_{62} \mathrm{O}_{16}$ & Grr \\
\hline 30. & 27.67 & $515.19080^{\mathrm{a}}(-0.38)$ & Limonin & $\mathrm{C}_{26} \mathrm{H}_{30} \mathrm{O}_{8}$ & Dc \\
\hline 31. & 28.26 & $483.16458(-0.93)$ & Limonin diosphenol & $\mathrm{C}_{26} \mathrm{H}_{28} \mathrm{O}_{9}$ & Dc \\
\hline 32. & 30.70 & $453.19064(-0.69)$ & Obacunone $^{\mathrm{b}}$ & $\mathrm{C}_{26} \mathrm{H}_{30} \mathrm{O}_{7}$ & Dc \\
\hline
\end{tabular}

${ }^{\mathrm{a}}[\mathrm{M}+\mathrm{HCOO}]-;{ }^{\mathrm{b}}$ compared with the standard compound. Ff: Forsythiae Fructus; Grr: Glycyrrhizae Radix et Rhizoma; Dc: Dictamni Cortex; Ir: Imperatae Rhizoma; Am: Atractylodis Macrocephalae; Dr: Dioscoreae Rhizoma; Pr: Pseudostellariae Radix; Po: Poria.

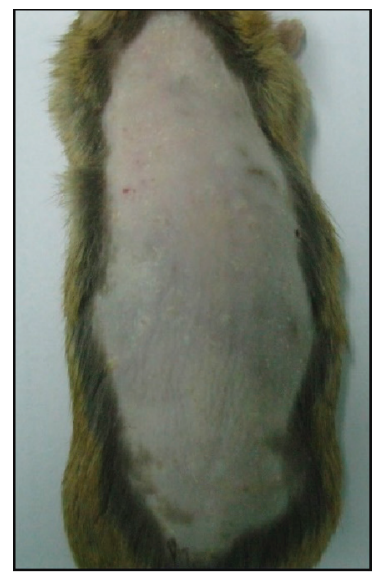

Control

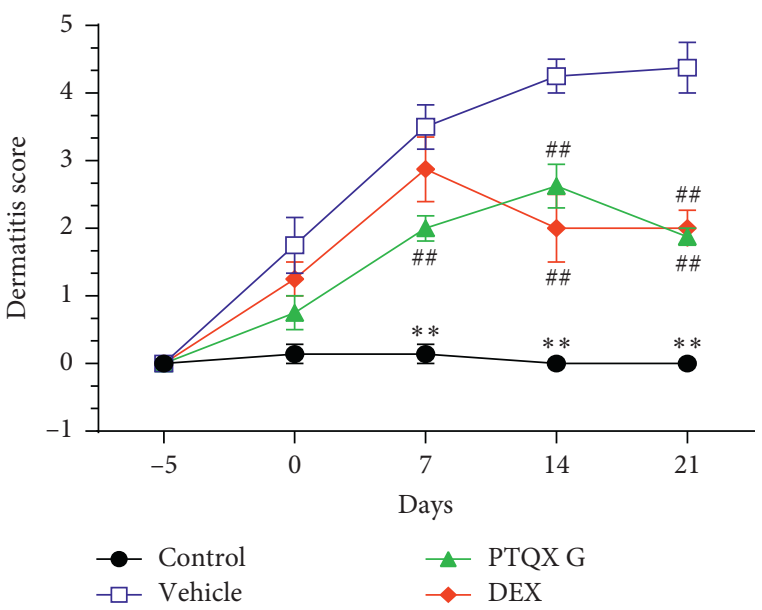

(b)

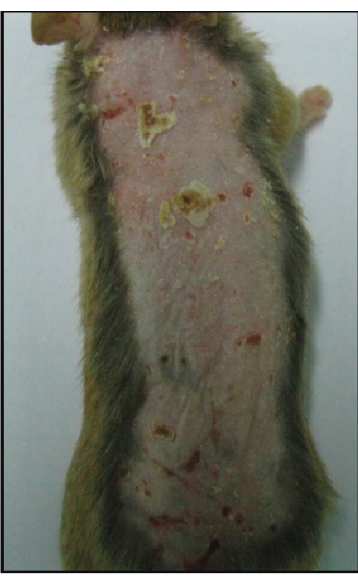

Vehicle (a)

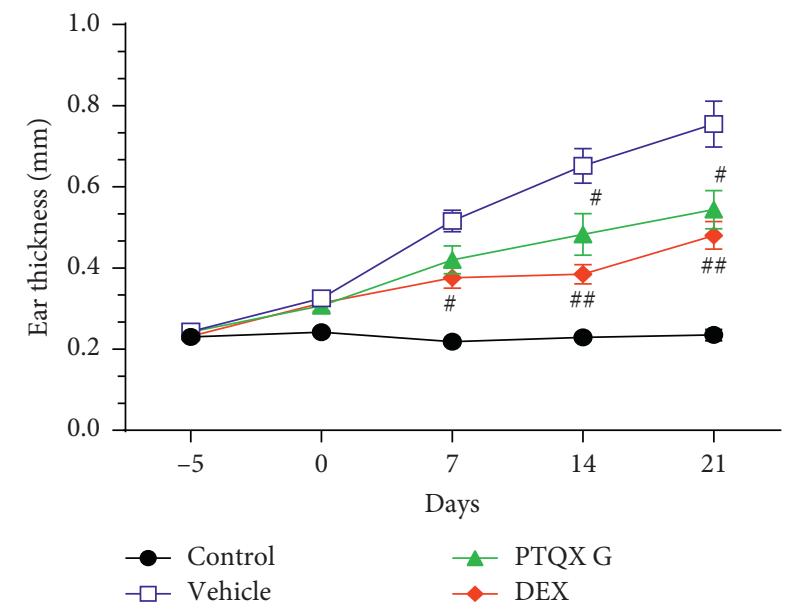

(c)

FIGURE 3: Continued. 


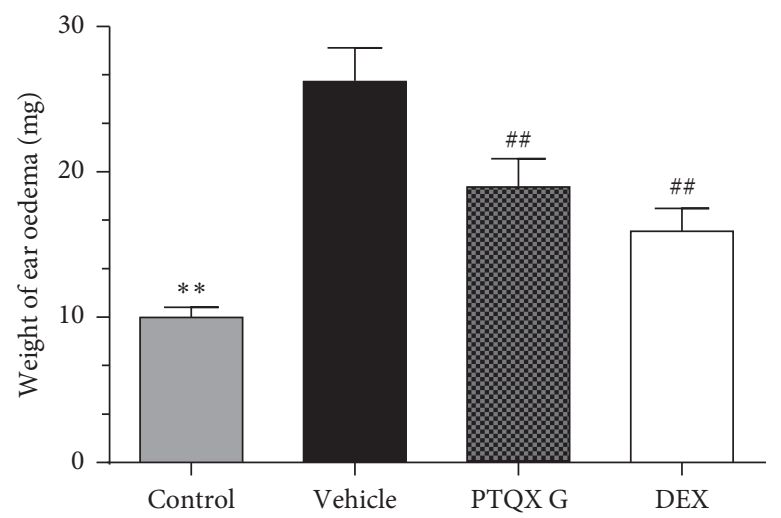

(d)

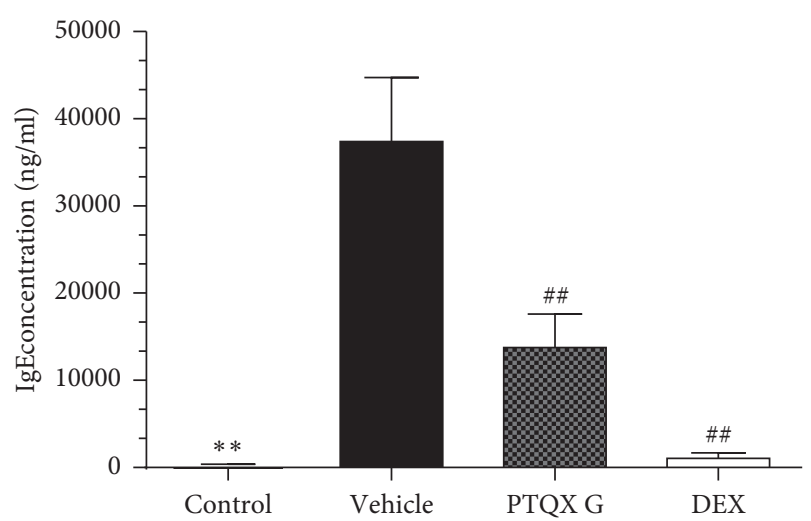

(e)

FIgURE 3: Treatment with PTQX inhibits 1-chloro-2,4-dinitrobenzene- (DNCB-) induced atopic dermatitis- (AD-) like skin inflammation in $\mathrm{NC} / \mathrm{Nga}$ mice. (a). Representative photographs taken on day 7. (b). Dermatitis scores were evaluated weekly from day -5 to day 21 . (c). Ear thickness was measured from day -5 to 21. (d). The effect of PTQX on the ear weights of NC/Nga mice on day 21. (e). PTQX reduces the serum levels of total IgE in NC/Nga mice. The results are expressed as means \pm standard errors of the means for 7-8 mice per group. Control, untreated group; vehicle, DNCB-induced group; PTQX, PTQX-treated group; and DEX, dexamethasone-treated group. Statistically significant differences with respect to the control and vehicle groups are expressed as ${ }^{*} P<0.05,{ }^{* *} P<0.01$ and ${ }^{\#} P<0.05$, ${ }^{\# \#} P<0.01$, respectively.
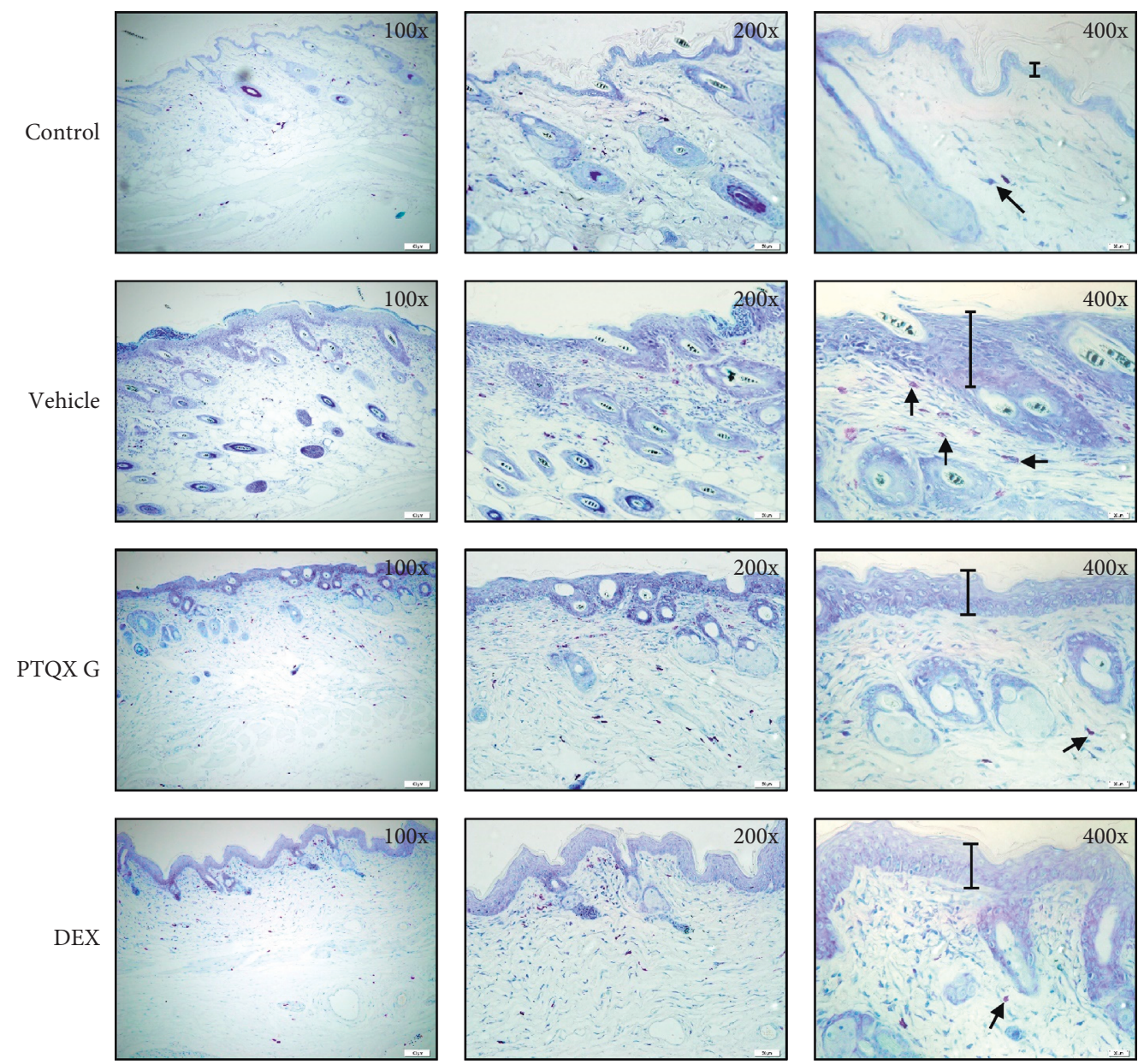

(a)

FIGURE 4: Continued. 


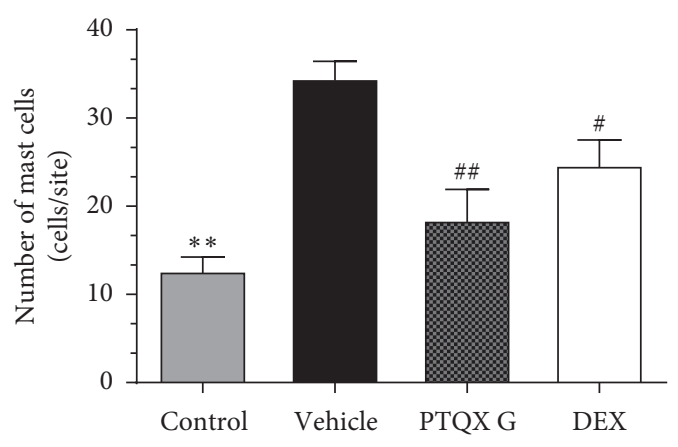

(b)

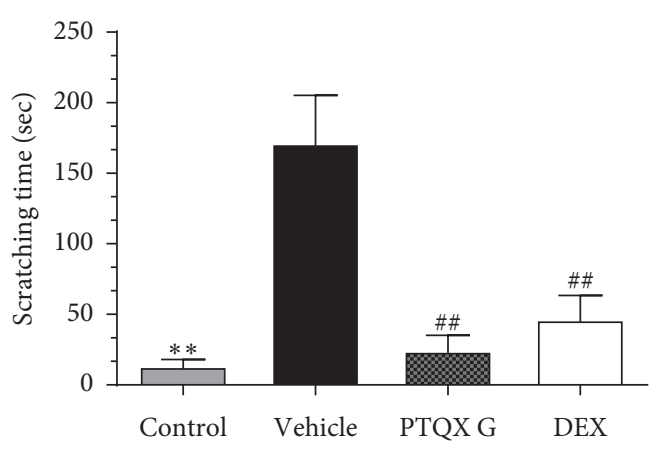

(c)

FIGURE 4: Effect of PTQX on 1-chloro-2,4-dinitrobenzene- (DNCB-) induced mast cell infiltration and scratching frequency in the dermal lesions of NC/Nga mice. (a) Representative images depicting the histological features of skin samples collected on day 21. Toluidine blue (TB) staining was used to identify mast cells (arrows). Cells were counted under a microscope at 100x, 200x, and 400x magnification. (b) Mast cells were counted in five randomly selected sites of TB-stained sections. (c) Effect of PTQX on the DNCB-induced scratching incidence in NC/Nga mice. The scratching time was evaluated on the penultimate day of the experiment (day 20). The results are expressed as the means \pm standard errors of the means of 3 mice. Statistically significant differences with respect to the control and vehicle groups are expressed as ${ }^{*} P<0.05,{ }^{* *} P<0.01$ and ${ }^{\#} P<0.05$, ${ }^{\# \#} P<0.01$, respectively.

scratching behaviour could potentially be used to evaluate the antipruritic efficacy of PTQX in a NC/Nga mouse model of $\mathrm{AD}$ [24]. On day 20, we carefully monitored the animals for $20 \mathrm{~min}$ and quantified the scratching time, defined as the time spent rubbing their ears, nose, and dorsal skin with their hind paws. DNCB-induced NC/Nga mice (vehicle) exhibited the longest scratching time, $171.2 \pm 34.1 \mathrm{~s}$. Comparatively, the PTQX group had a significantly shorter scratching time of $24 \pm 11.12 \mathrm{~s}$, an approximately $86 \%$ reduction. Treatment with DEX also reduced the DNCB-induced scratching time to $46.25 \pm 17.24 \mathrm{~s}$ (Figure $4(\mathrm{c})$ ).

3.5. PTQX Inhibited DNCB-Induced AD-Like Skin Inflammation in NC/Nga Mice. To determine whether PTQX could inhibit $\mathrm{AD}$ in NC/Nga mice, we used DNCB to induce $\mathrm{AD}$-like skin inflammation in the presence or absence of PTQX. Histologically, PTQX treatment significantly reduced the epidermal thickness, inflammatory cell infiltration, and dense fibrous bundles in the ears of model mice (Figure 5(a)). PTQX treatment also markedly reduced the infiltration of lymphocytes, inflammatory cells, and mononuclear cells into dorsal skin lesions (Figure 5(b)). Furthermore, IHC revealed that the ears of mice with DNCB-induced swelling were more strongly stained with CD4- and CD8-specific antibodies, compared to the vehicle mice. The populations of CD8+ and CD4+ Tcells in the ears also decreased significantly after treatment with PTQX (Figure 5(c) and Figure 5(d)), confirming that this formula attenuated the cutaneous inflammation induced by DNCB in NC/Nga mice.

3.6. PTQX Regulates CD4+ T-Cell Proliferation and Differentiation. Our interest in and understanding of the importance of $\mathrm{CD} 4+\mathrm{T}$ cells in the development of $\mathrm{AD}$ have increased in recent years [25]. Upon activation, CD4+ T cells mainly differentiate into four subsets: Th1, Th2, Th17, and Treg. Studies of Th1/Th2 and Th17/Treg differentiation patterns during the progression of $\mathrm{AD}$ indicate the importance of CD4+ cells in this disease $[5,26]$. Therefore, we focused on the effect of PTQX on the contributions of $\mathrm{CD} 4+\mathrm{T}$ cells to immunomodulation in AD. Interestingly, our results showed that PTQX had almost no effect on CD4+ T-cell differentiation in the spleen (Figure 6(a)). In the lymph nodes, however, PTQX significantly inhibited the proliferation of CD4+ IL-4 and CD4+ Foxp3 Treg cells, compared to vehicle treatment (Figure 6(b)). Our study partly explains the mechanism underlying the pharmacological effects of PTQX. Specifically, this formula regulates the Th17/Treg balance to inhibit the development of AD. Our results indicate that PTQX suppressed the symptoms of DNCB-induced $\mathrm{AD}$ by modulating CD4+ T-cell proliferation and differentiation and particularly by regulating the Th17/Treg balance.

\section{Discussion}

Various studies have long demonstrated the clinical effects of TCM formulas and related treatments for AD [27]. In TCM, Si Wan Feng described a condition comparable to atopic eczema/AD and the treatment of this disease requires the differentiation of the chief syndrome and an analysis and determination of treatment strategies. In TCM, treatment aims to strengthen the body against disease and strongly emphasises Qi (i.e., vital energy) and the Yin-Yang balance (i.e., negative and positive equilibrium) as factors contributing to health and well-being. According to TCM theory, the aetiology of $\mathrm{AD}$ is closely related to "wind," "dampness," and "heat" [28] and traditional formulas can cleanse and clear these factors. Therefore, TCM is intended to improve a person's general health and wellness while treating specific diseases or common ailments. However, more high-quality RCTs are needed to assess the efficacy of TCM. The Chinese herbal formula PTQX is intended to simultaneously invigorate the spleen while reducing dampness, clearing heartfire, and relieving pruritus. 

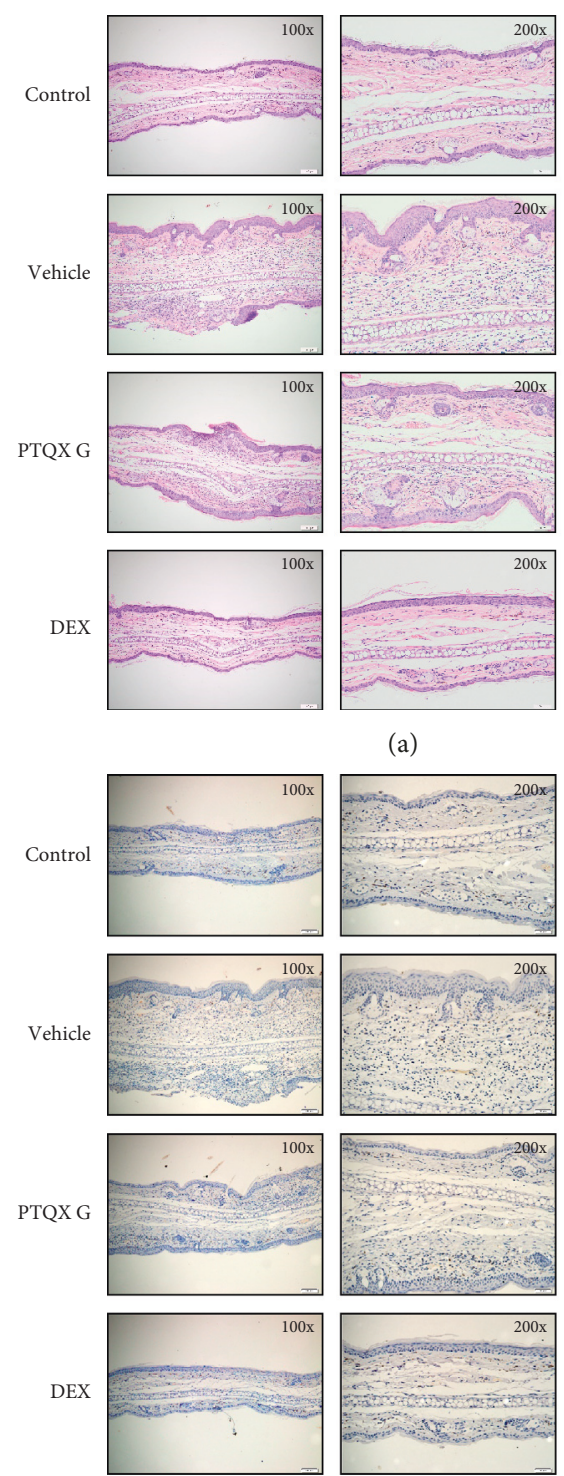

(a)
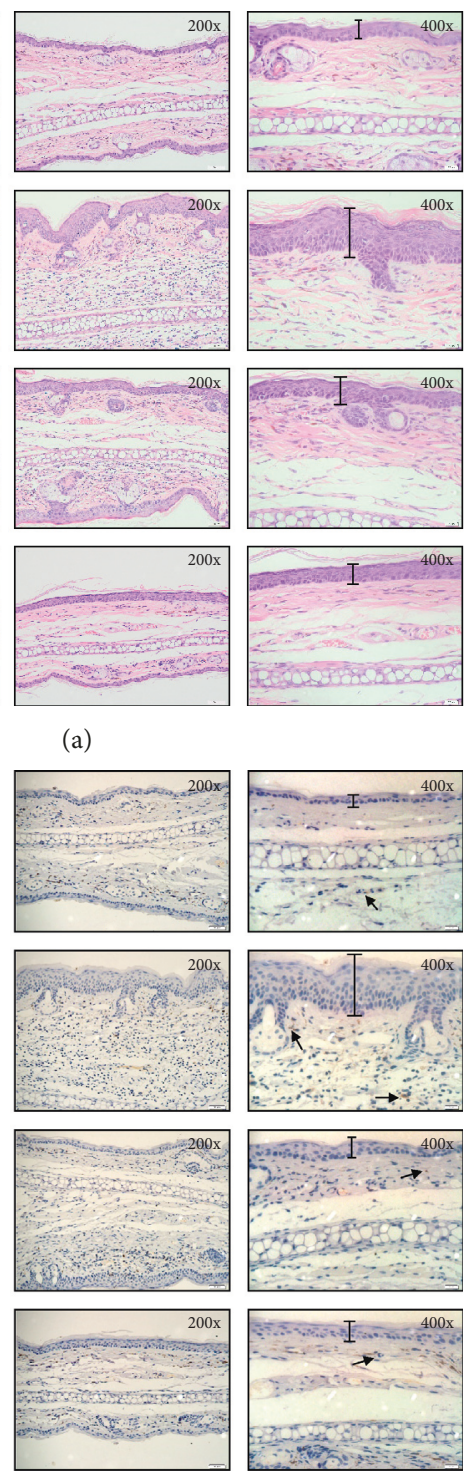

(c)
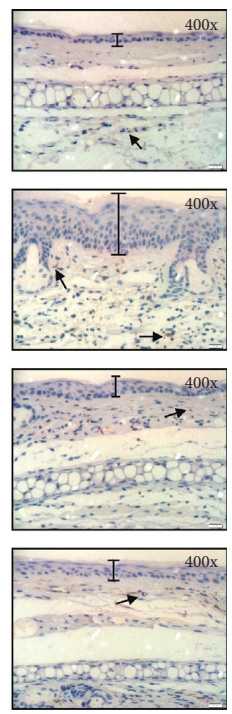
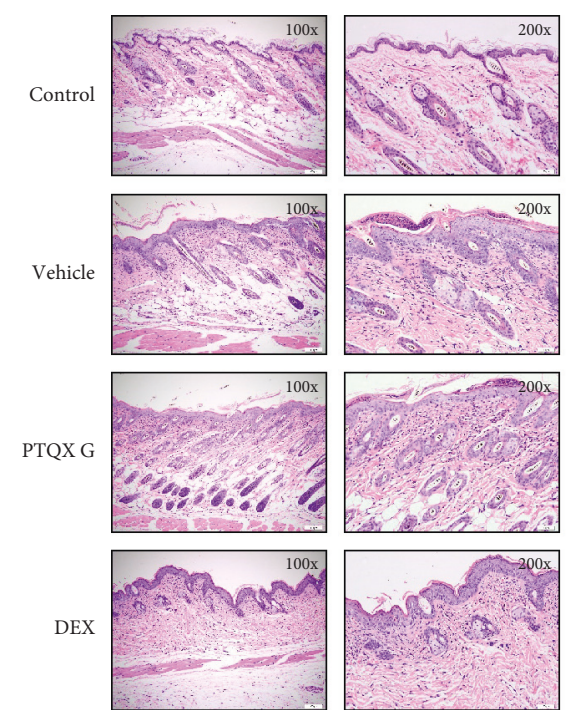

(b)
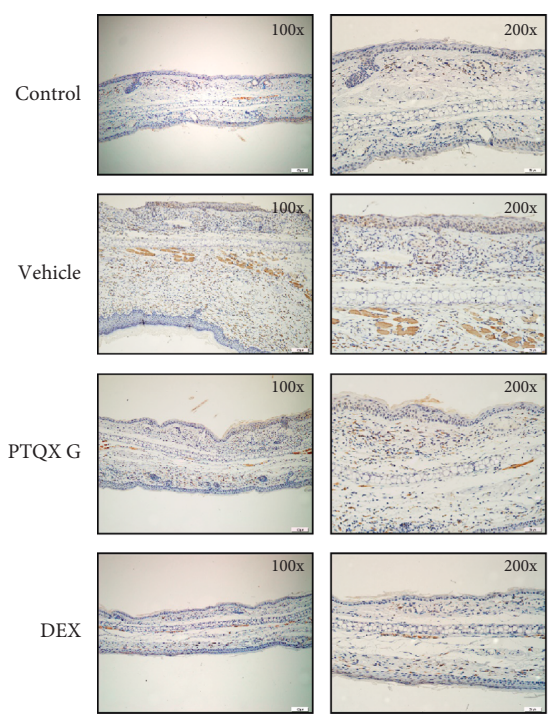

(d)
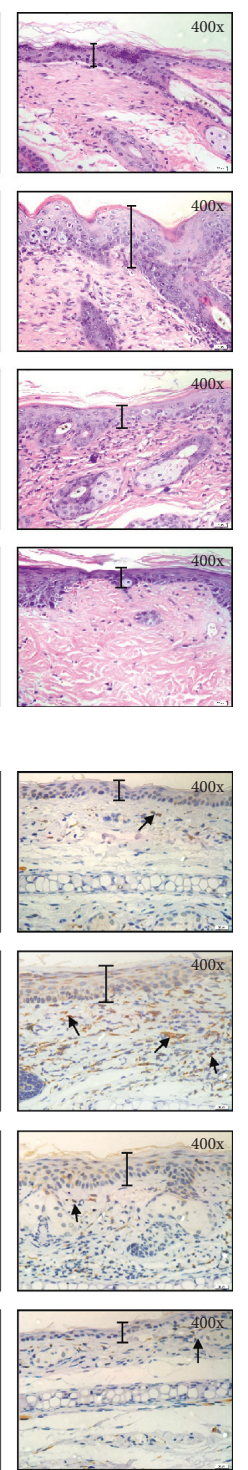

FIgURE 5: PTQX suppresses tissue inflammation and immune cell infiltration in NC/Nga mice. Atopic dermatitis was induced by the repeated topical application of 1-chloro-2,4-dinitrobenzene (DNCB), with or without PTQX treatment. Sections of the ears (a) and dorsal skin (b) were stained with haematoxylin and eosin (HE). The ear tissues were also subjected to an immunohistochemical analysis of CD4+ (c) and CD8+ (d) T lymphocytes to estimate epidermal inflammation (arrows). A representative example of 3 independent experiments is shown.

In 2015, we reported the results of an assessor-blind, multicentre RCT of PTQX for the treatment of $\mathrm{AD}[9,29]$. Still, the molecular mechanism underlying the effects of PTQX against $\mathrm{AD}$ urgently requires attention. In this study, we found that PTQX could improve the AD-like symptoms of redness, eczema, and pruritus induced by $\mathrm{DNCB}$ in $\mathrm{NC} /$ Nga mice. Our previous experimental and clinical study also demonstrated that PTQX was an effective treatment for AD. Therefore, it would be helpful to further clarify the mechanism of action of PTQX.

To date, several murine models of $\mathrm{AD}$ have been established, including transgenic, NC/Nga, NOA, C57BL/6, and $\mathrm{BALB} / \mathrm{c}$ mice [30-33]. Although previous studies have used animal models displaying some of the characteristic histopathological features of $\mathrm{AD}$, the disease pathogenesis and mechanisms remain incompletely understood. This disease is characterised by the infiltration of macrophage and inflammatory cells and Langerhans cells into the dermis, activation of $\mathrm{T}$ lymphocytes and dendritic cells (DCs), and the proliferation and activation of mast cells [34]. Our present study, based on NC/Nga AD model mice, provides important information about the causes of the basic and clinical AD-like symptoms induced by DNCB. Mast cells are critical to the induction of allergic diseases such as asthma, allergic rhinitis, and $\mathrm{AD}$ [35]. Here, our findings suggest that PTQX suppresses the inflammatory infiltration of mast cells, which are thought to contribute to the pathogenesis of $\mathrm{AD}$. Our results indicate that PTQX can reduce the infiltration of 

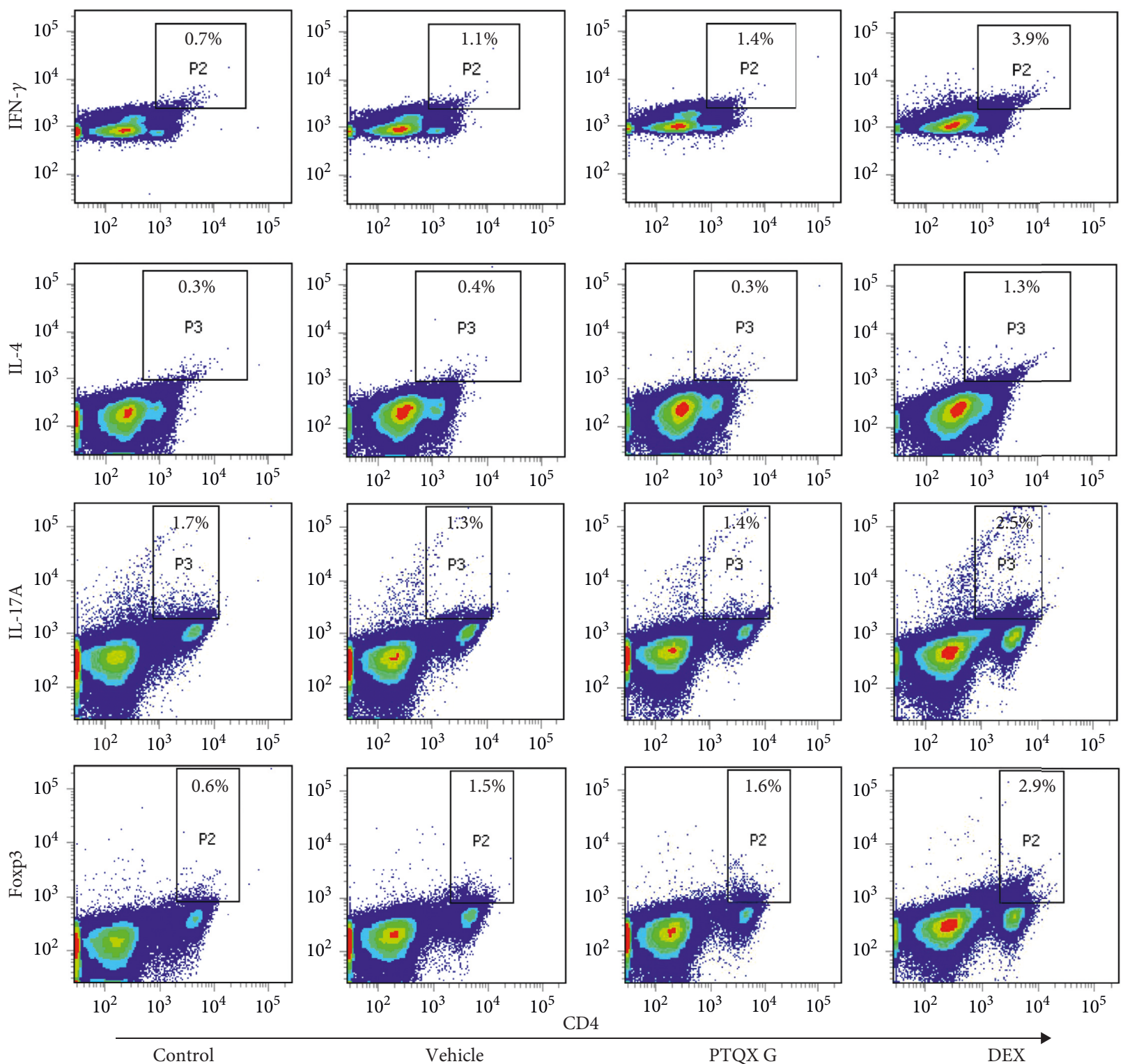

(a)

FIGURE 6: Continued. 

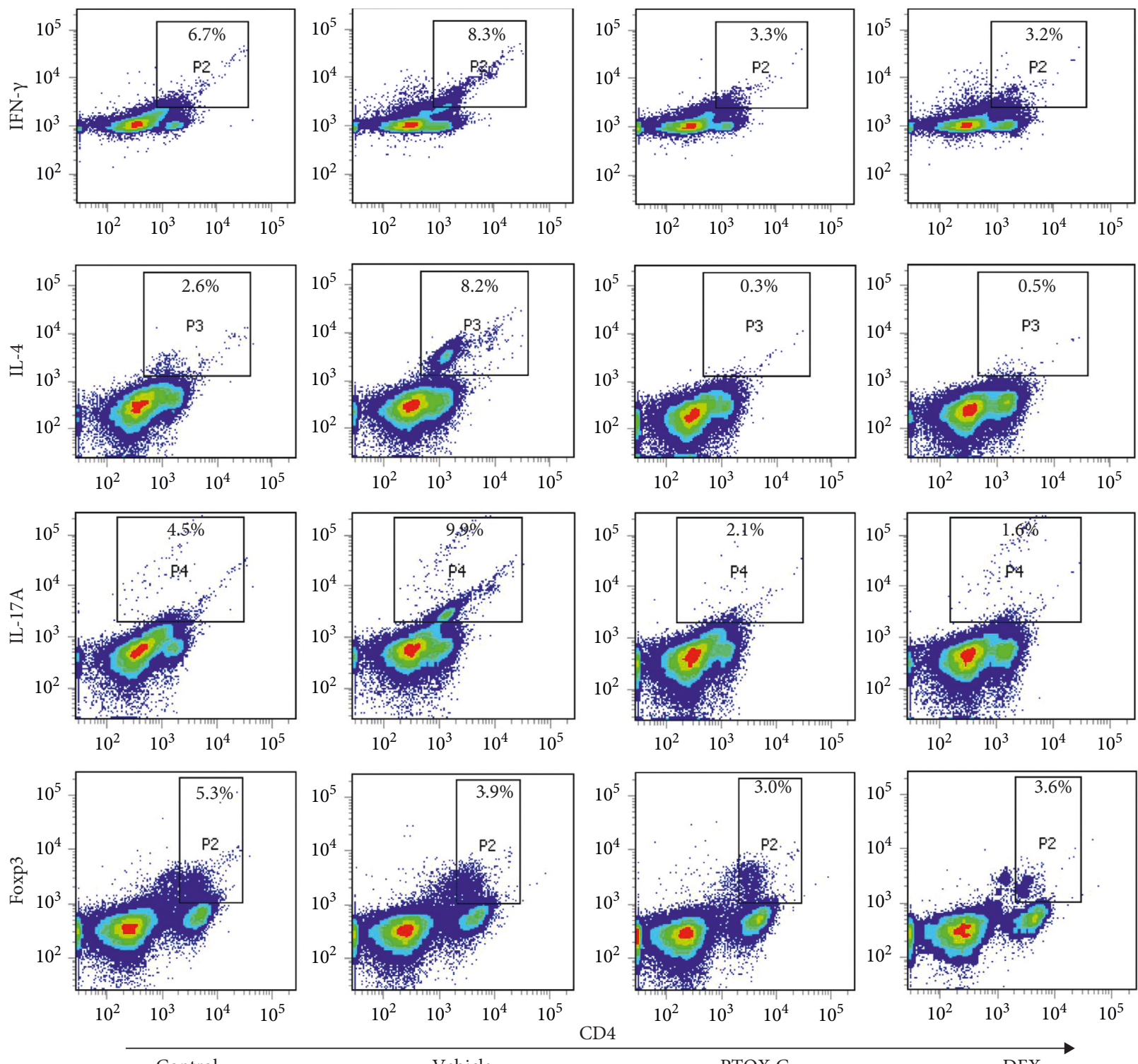

(b)

FIgURE 6: Treatment with PTQX inhibits the differentiation of T-helper 2 (Th2) and Th17 cells in the draining lymph nodes of NC/Nga mice with 1-chloro-2,4-dinitrobenzene- (DNCB-) induced atopic dermatitis. CD4+ Th1, Th2, Th17, and regulatory $\mathrm{T}$ (Treg) lymphocyte populations were selected by costaining for IFN- $\gamma$, IL-4, IL-17A, and Foxp3, respectively. Flow cytometry was used to evaluate these populations of lymphocytes in the spleens (a) and lymph nodes (b) of mice. A representative example of 2 replicate independent experiments is shown.

mast cells into skin lesions and alleviate the symptoms of $\mathrm{AD}$.

We further found that PTQX could inhibit the increases in epidermal and dermal thickness and serum IgE production observed in $\mathrm{AD}$ model mice. We were particularly interested in the immune regulatory effects of PTQX on the balance between the Th1, Th2, Th17, and Treg subtypes in the spleen and lymph nodes. Recent research has suggested that the additional activation of the Th22-, Th17/IL-23-, and Th1-related cytokine pathways depends on the subtype of the disease [36], while Th2 cells have been implicated in $\mathrm{AD}$ pathogenesis and development [37]. A skewed Th17/Treg balance may also play a vital role in several autoimmune, inflammatory, and allergic reactions. Nevertheless, the possible contribution of the Th17/Treg imbalance to $\mathrm{AD}$ remains unclear and further studies are needed. Our results suggest that PTQX treatment reduced the populations of Th1 and Th2 cells and regulated the ratio of Th17/Treg cells in DNCBtreated NC/Nga mice. Accordingly, treatment with PTQX may improve the Th17/Treg immune balance and help to regulate inflammation. 


\section{Conclusion}

Currently, AD cannot be fully cured or controlled. Our previous clinical studies have shown that PTQX can effectively reduce the disease severity and improve the quality of life and self-assessment of patients with moderate-to-severe AD. This study further shows that therapeutic effects of PTQX on AD by regulating Th2/Th17 cell balance and suppressing mast cell infiltration while effectively relieving pruritis and reducing the inflammatory response in a $\mathrm{NC} /$ Nga mouse model. Our results suggest that PTQX might be a good candidate for the treatment of $\mathrm{AD}$.

\section{Abbreviations}

AD: $\quad$ Atopic dermatitis

AO: Acetone-olive oil

DCs: Dendritic cells

DEX: Dexamethasone

DNCB: 1-Chloro-2,4-dinitrobenzene

ELISA: Enzyme-linked immunosorbent assay

HE: Haematoxylin and eosin

HPLC: High-performance liquid chromatography

ESI: Electrospray ionisation

IgE: Immunoglobulin $\mathrm{E}$

IHC: Immunohistochemistry

PMA: Phorbol-12-myristate-13-acetate

PTQX: Chinese herbal formula Pei Tu Qing Xin

QOL: Quality of life

SEM: Standard error of mean

TCM: Traditional Chinese medicine

TB: $\quad$ Toluidine blue

Th: T-helper cell type

Treg: Regulatory $\mathrm{T}$ cell.

\section{Data Availability}

The data used to support the findings of this study are included within the article.

\section{Conflicts of Interest}

The authors declare that they have no conflicts of interest.

\section{Authors' Contributions}

Dacan Chen designed the experiments, supervised all research, and revised the manuscript. Fenggen Yan conducted the experiments and wrote the text of the main manuscript. Jing Zhang, Xiong Li, Xiumei Mo, Junfeng Liu, Siqi Ye, Ying Lin, Xiaohui Qiu, and Simon Mingyuen Lee conducted the experiments and contributed to the interpretation of data. All authors reviewed the results and approved the final version.

\section{Acknowledgments}

This work was supported by the National Natural Science Foundation of China (project code: 81774307), the Department of Finance of Guangdong Province (Yue cai gong
[2016] no. 150), the Specific Research Fund for TCM Science and Technology of Guangdong Provincial Hospital of Chinese Medicine (project code: YN2015MS06, YN2015QN09, and YN2016QJ11), the Traditional Chinese Medicine Bureau of Guangdong Province (project code: 20183004), and the Department of Science and Technology of Guangdong Province (grant no. (2017)105; project code: 2017A030310122).

\section{References}

[1] R. Sidbury, D. M. Davis, D. E. Cohen et al., "Guidelines of care for the management of atopic dermatitis: section 3. Management and treatment with phototherapy and systemic agents," Journal of the American Academy of Dermatology, vol. 71, no. 2, pp. 327-349, 2014.

[2] S. Weidinger and N. Novak, "Atopic dermatitis," The Lancet, vol. 387, no. 10023, pp. 1109-1122, 2016.

[3] K. Hon, B. Chan, and P. Leung, "Chinese herbal medicine research in eczema treatment," Chinese Medicine, vol. 6, no. 17, pp. 1-8, 2011.

[4] T. Bieber, "Atopic dermatitis," New England Journal of Medicine, vol. 358, no. 14, pp. 1483-1494, 2008.

[5] C. Su, T. Yang, Z. Wu et al., "Differentiation of T-helper cells in distinct phases of atopic dermatitis involves Th1/Th2 and Th17/Treg," European Journal of Inflammation, vol. 15, no. 1, pp. 46-52, 2017.

[6] K. Eyerich and N. Novak, "Immunology of atopic eczema: overcoming the Th1/Th2 paradigm," Allergy, vol. 68, no. 8, pp. 974-982, 2013.

[7] L. P. Ngoc, D. R. Gold, A. O. Tzianabos, S. T. Weiss, and J. C. Celedón, "Cytokines, allergy, and asthma," Current Opinion in Allergy and Clinical Immunology, vol. 5, no. 2, pp. 161-166, 2005.

[8] L. Ma, H.-B. Xue, X.-H. Guan et al., "The Imbalance of Th17 cells and $\mathrm{CD}^{+} \mathrm{CD} 25^{\text {high }} \mathrm{Foxp} 3^{+}$Treg cells in patients with atopic dermatitis," Journal of the European Academy of Dermatology and Venereology, vol. 28, no. 8, pp. 1079-1086, 2014.

[9] J. Liu, X. Mo, D. Wu et al., "Efficacy of a Chinese herbal medicine for the treatment of atopic dermatitis: a randomised controlled study," Complementary Therapies in Medicine, vol. 23, no. 5, pp. 644-651, 2015.

[10] S. X. Gu, A. L. Zhang, M. E. Coyle et al., "Chinese herbal medicine granules (PTQX) for children with moderate to severe atopic eczema: study protocol for a randomised controlled trial," Trials, vol. 16, no. 294, pp. 1-6, 2015.

[11] X. Mo, J. Liu, and Y. Chen, "Pharmacodynamic research of peitu qingxin keli in relieving itching of experimental itchy skin Guinea pig model," Journal of New Chinese Medicine, vol. 47, no. 7, pp. 272-274, 2018.

[12] X. Mo, J. Liu, Y. Chen et al., "Pharmacodynamic research of the anti-inflammation effect on PeiTu QingXin (PTQX) granules," Journal of Basic Chinese Medicine, vol. 21, no. 6, pp. 744-746, 2015.

[13] Y. Lin, W. Meng, X. Zhang et al., "Effect of peitu qingxin granules on Th1/Th2 cells in spleen of atopic dermatitis mice mode," Journal of New Chinese Medicine, vol. 49, no. 6, pp. 5-9, 2017.

[14] J. K. Choi and S.-H. Kim, "Rutin suppresses atopic dermatitis and allergic contact dermatitis," Experimental Biology and Medicine, vol. 238, no. 4, pp. 410-417, 2013. 
[15] H. Yu, H. Li, Y. Li, M. Li, and G. Chen, "Effect of isoliquiritigenin for the treatment of atopic dermatitis-like skin lesions in mice," Archives of Dermatological Research, vol. 309, no. 10, pp. 805-813, 2017.

[16] Y. Wang, Y. Zhang, G. Peng, and X. Han, "Glycyrrhizin ameliorates atopic dermatitis-like symptoms through inhibition of HMGB1," International Immunopharmacology, vol. 60, pp. 9-17, 2018.

[17] H. Kim, J. R. Kim, H. Kang et al., “7,8,4'-trihydroxyisoflavone attenuates DNCB-induced atopic dermatitis-like symptoms in NC/nga mice," PLoS ONE, vol. 9, no. 8, Article ID e104938, 2014.

[18] D. Y. M. Leung, R. L. Hirsch, L. Schneider et al., "Thymopentin therapy reduces the clinical severity of atopic dermatitis," Journal of Allergy and Clinical Immunology, vol. 85, no. 5, pp. 927-933, 1990.

[19] L. Sun, H. Wei, F. Zhang et al., "Qualitative analysis and quality control of Traditional Chinese Medicine preparation Tanreqing injection by LC-TOF/MS and HPLC-DAD-ELSD," Analytical Methods, vol. 5, no. 22, pp. 6431-6440, 2013.

[20] H. Guo, A.-H. Liu, M. Ye, M. Yang, and D.-A. Guo, "Characterization of phenolic compounds in the fruits of Forsythia suspensa by high-performance liquid chromatography coupled with electrospray ionization tandem mass spectrometry," Rapid Communications in Mass Spectrometry, vol. 21, no. 5, pp. 715-729, 2007.

[21] Y. Liu, J. Yang, and Z. Cai, "Chemical investigation on Sijunzi decoction and its two major herbs Panax ginseng and Glycyrrhiza uralensis by LC/MS/MS," Journal of Pharmaceutical and Biomedical Analysis, vol. 41, no. 5, pp. 1642-1647, 2006.

[22] E. Azimi, V. B. Reddy, and E. A. Lerner, "MRGPRX2, atopic dermatitis, and red man syndrome," Itch, vol. 2, no. 1, p. e5, 2017.

[23] T. Kawakami, T. Ando, M. Kimura, B. S. Wilson, and Y. Kawakami, "Mast cells in atopic dermatitis," Current Opinion in Immunology, vol. 21, no. 6, pp. 666-678, 2009.

[24] N. Takano, I. Arai, and M. Kurachi, "Analysis of the spontaneous scratching behavior by NC/Nga mice: a possible approach to evaluate antipruritics for subjects with atopic dermatitis," European Journal of Pharmacology, vol. 471, no. 3, pp. 223-228, 2003.

[25] J. H. Lee, Y. S. Lee, E.-J. Lee, J. H. Lee, and T.-Y. Kim, "Capsiate inhibits DNFB-induced atopic dermatitis in NC/ nga mice through mast cell and $\mathrm{CD}_{4+}$ T-cell inactivation," Journal of Investigative Dermatology, vol. 135, no. 8, pp. 1977-1985, 2015.

[26] N.-H. Jeong, E.-J. Yang, M. Jin et al., "Esculetin from Fraxinus rhynchophylla attenuates atopic skin inflammation by inhibiting the expression of inflammatory cytokines," International Immunopharmacology, vol. 59, pp. 209-216, 2018.

[27] Z.-f. Shi, T.-b. Song, J. Xie, Y.-q. Yan, and Y.-p. Du, "The traditional Chinese medicine and relevant treatment for the efficacy and safety of atopic dermatitis: a systematic review and meta-analysis of randomized controlled trials," EvidenceBased Complementary and Alternative Medicine, vol. 2017, p. 20, 2017.

[28] X. Lin, C. Tu, and C. Yang, "Study on treatment of eczema by Chinese herbal medicine with anti-type IV allergic activity," Chinese Journal of Integrated Traditional and Western Medicine, vol. 20, no. 4, pp. 258-260, 2000

[29] S. X. Gu, X. Mo, A. L. Zhang et al., "A Chinese herbal medicine preparation (Pei Tu Qing Xin) for children with moderate-tosevere atopic eczema: a pilot randomized controlled trial,"
British Journal of Dermatology, vol. 179, no. 6, pp. 1404-1405, 2018.

[30] J. Yoo, M. Omori, D. Gyarmati et al., "Spontaneous atopic dermatitis in mice expressing an inducible thymic stromal lymphopoietin transgene specifically in the skin," The Journal of Experimental Medicine, vol. 202, no. 4, pp. 541-549, 2005.

[31] K. Natori, M. Tamari, O. Watanabe et al., "Mapping of a gene responsible for dermatitis in NOA (Naruto Research Institute Otsuka Atrichia) mice, an animal model of allergic dermatitis," Journal of Human Genetics, vol. 44, no. 6, pp. 372-376, 1999.

[32] H. Matsuda, N. Watanabe, G. P. Geba et al., "Development of atopic dermatitis-like skin lesion with IgE hyperproduction in NC/Nga mice," International Immunology, vol. 9, no. 3, pp. 461-466, 1997.

[33] H. Jin, R. He, M. Oyoshi, and R. S. Geha, "Animal models of atopic dermatitis," Journal of Investigative Dermatology, vol. 129, no. 1, pp. 31-40, 2009.

[34] S. Kasraie and T. Werfel, "Role of macrophages in the pathogenesis of atopic dermatitis," Mediators Inflamm, vol. 2013, Article ID 942375, 15 pages, 2013.

[35] K. Amin, "The role of mast cells in allergic inflammation," Respiratory Medicine, vol. 106, no. 1, pp. 9-14, 2012.

[36] P. M. Brunner, E. Guttman-Yassky, and D. Y. M. Leung, "The immunology of atopic dermatitis and its reversibility with broad-spectrum and targeted therapies," Journal of Allergy and Clinical Immunology, vol. 139, no. 4, pp. S65-S76, 2017.

[37] A.-x. Wang and N. X. Landén, "New insights into T cells and their signature cytokines in atopic dermatitis," IUBMB Life, vol. 67, no. 8, pp. 601-610, 2015. 


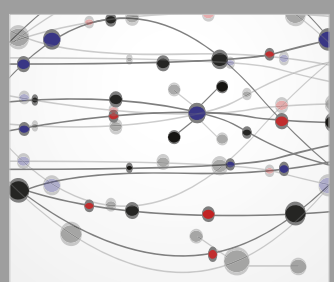

The Scientific World Journal
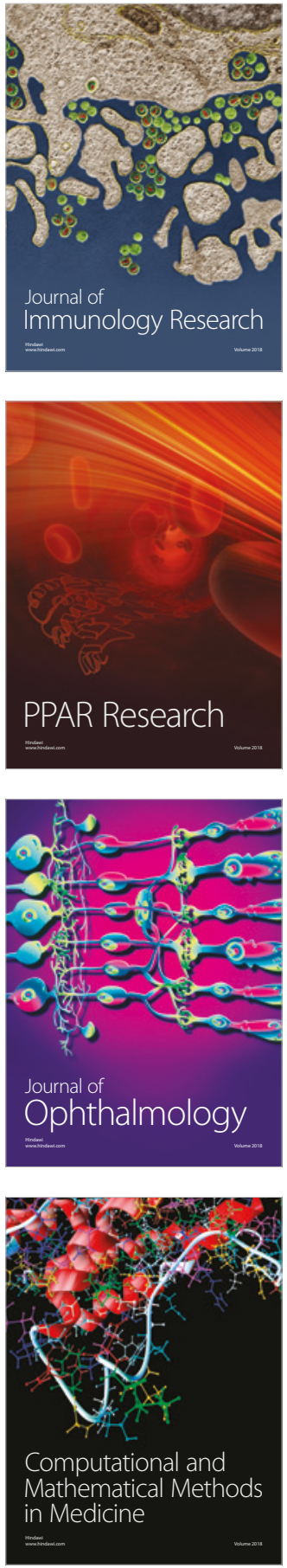

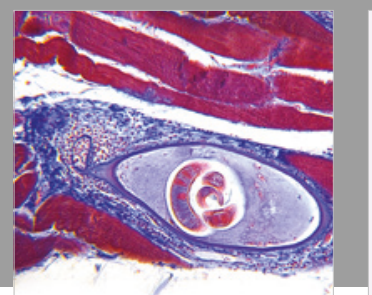

Gastroenterology Research and Practice

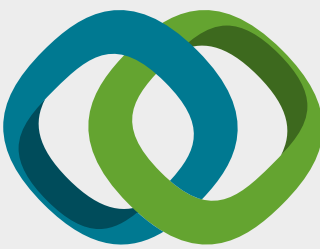

\section{Hindawi}

Submit your manuscripts at

www.hindawi.com
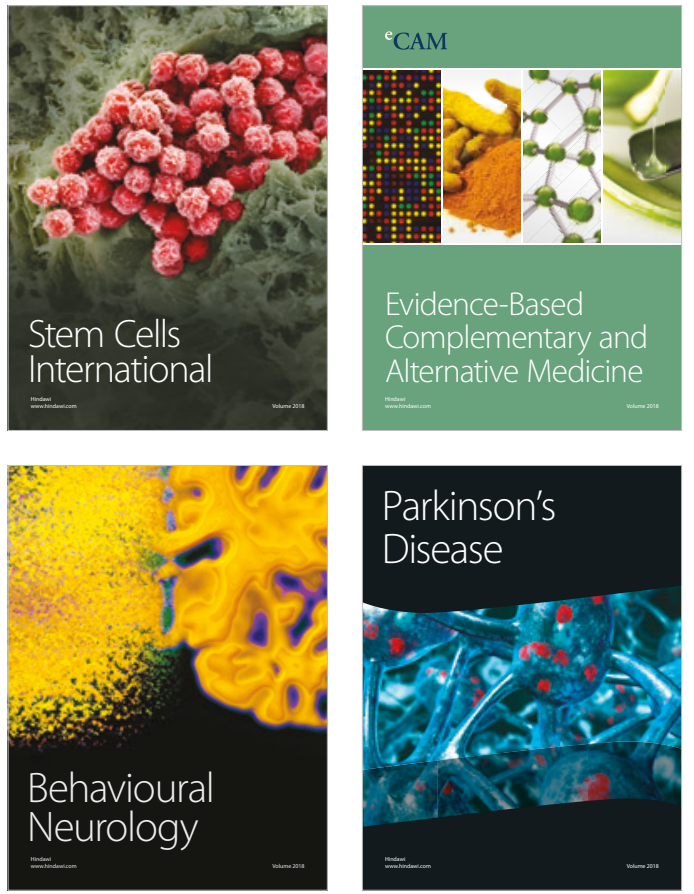

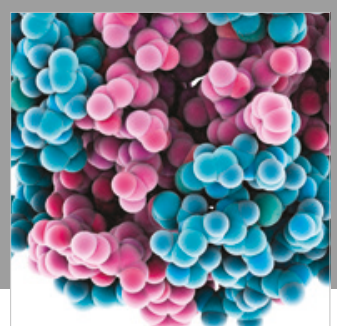

ournal of

Diabetes Research

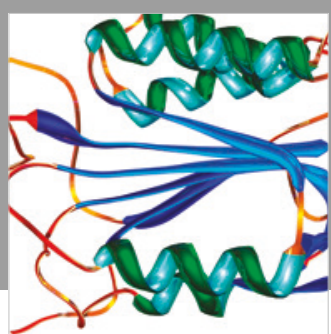

Disease Markers
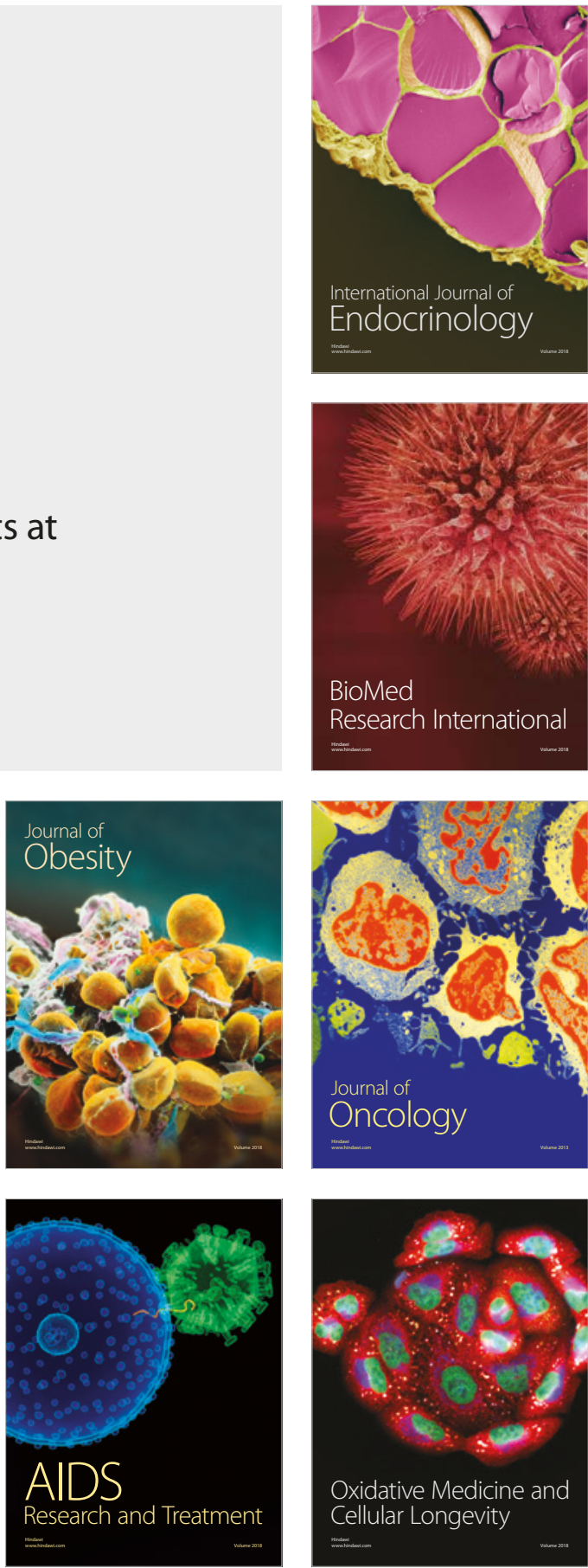